\title{
Genre et migrations dans les études atlantiques de 1500 à nos jours
}

Spatializing Gender and Migration: The Periodization of Atlantic Studies, 1500-

Present

Género y migraciones en los estudios atlánticos del año 1500 a nuestros días

Donna R. Gabaccia

\section{(2) OpenEdition}

Journals

Édition électronique

URL : https://journals.openedition.org/remi/7159

DOI : $10.4000 /$ remi.7159

ISSN : $1777-5418$

Éditeur

Université de Poitiers

Édition imprimée

Date de publication : 1 janvier 2015

Pagination : 15-37

ISBN : 979-10-90426-24-5

ISSN : 0765-0752

Référence électronique

Donna R. Gabaccia, « Genre et migrations dans les études atlantiques de 1500 à nos jours », Revue européenne des migrations internationales [En ligne], vol. 31 - n¹ | 2015, mis en ligne le 01 janvier 2018, consulté le 14 avril 2022. URL : http://journals.openedition.org/remi/7159; DOI : https://doi.org/ 10.4000/remi.7159 


\section{Genre et migrations dans les études atlantiques de 1500 à nos jours}

\section{Donna R. Gabaccia ${ }^{1}$}

Pour les spécialistes des études de genre et des recherches féministes, qui travaillent notamment dans les champs des études atlantiques et des Global Studies, la dernière décennie est assez paradoxale. Ils ont cartographié des réseaux de connexions entre l'Atlantique et le reste du monde, mais personne n'a proposé une périodisation précise de l'intégration globale qui en découle (Gabaccia, 2004 ; Kupperman, 2012). Nous ignorons toujours pendant combien de temps l'Atlantique ${ }^{2}$ est resté une région à part et à quelle date l'intégration mondiale a pris plus d'importance que les systèmes macrorégionaux de circulation et d'échange. Par ailleurs, nous constatons avec surprise que, si des féministes comme Doreen Massey (1994) et Merry Wiesner-Hanks (2007) ont déploré le peu d'attention accordé au genre dans les Global Studies, les chercheurs en sciences sociales de leur côté ont souligné la "féminisation " des migrations en cours à l'échelle mondiale et mis l'accent sur la différence considérable entre les migrations d'après 1960, féminisées et intégrées mondialement, et celles d'avant 1945, traversant l'Atlantique et à dominante masculine (Silvey, 2006 ; Silvey et Lawson, 1999 ; Sinke 2006). La différence pourrait venir du fait que le passage à l'échelle mondiale a été accompagné à la fois par un nouveau rapport entre genre et migration et par la fin des migrations à dominante masculine. L' Atlantique demeure isolé jusqu'à la fin de la Seconde Guerre mondiale, ce qui justifierait une approche sur le temps long de I'histoire de I'Atlantique de 1500 jusqu'à un passé récent.

Dans les derniers travaux des historiens et des géographes des migrations, l'espace où se déplacent les migrants est étudié dans une perspective de genre: le local et les petites échelles sont souvent considérés comme étant féminins, domestiques et intimes, tandis que les échelles nationale et macrorégionale,

\footnotetext{
1 Professor of History, University of Toronto Scarborough, 1265 Military Trail, Room HW527 Toronto, Ontario, M1C 1A4 Canada; donna.gabaccia@utoronto.ca. Donna R. Gabaccia (2014) Spatializing Gender and Migration : The Periodization of Atlantic Studies, 1500 to the Present, Atlantic Studies : Global Currents, 11:1, 7-27, DOI:10.1080/1478 8810.2014.870702 (Article traduit par Manuel Benguigui avec I'autorisation de I'auteure).

2 « L'histoire atlantique ne se résume plus à l'étude d'un espace, d'un empire maritime et colonial, ou de liens bilatéraux entre deux pays de part et d'autre de l'océan ; elle constitue plutôt une approche conceptuelle, une grille de lecture : selon les termes d'Alan Karras, les atlanticistes considèrent ainsi que le monde atlantique constitue " a unit of analysis " qui permet d'appréhender la colonisation des Amériques d'une manière comparatiste, intégrée et transnationale dans une tentative d'histoire totale ou global history. " (Van Ruymbeke, 2008).
} 
ainsi que l'espace global, sont vus comme publics, masculins et traversés par des conflits (Castles et Miller, 2009). Pendant longtemps, les chercheurs ont supposé qu'une majorité de femmes et de jeunes filles n'effectuaient que des déplacements sur de courtes distances pour devenir "domestiques " tandis que chez les hommes prédominaient les déplacements internationaux sur une longue distance (Ravenstein, 1885 et 1889). Dès lors, les recherches se sont organisées selon la distance parcourue (King, Skeldon et Vullnetari, 2008). Un effort de théorisation et nombre de travaux empiriques ont mobilisé la notion de genre dans des échelles d'analyse plus vastes en précisant comment la création de nations et de diasporas exigeait d'imaginer les unes et les autres comme des espaces intimes, tandis que des rapports fondés sur l'amour familial hétéronormatif dans l'intimité ont rendu possible les solidarités nationale et nationaliste (Davis, 1997 ; Herzfeld, 2004). Les études sur l'Atlantique au début de l'époque moderne montrent également comment des chercheurs élargissent continuellement les analyses de genre à l'échelle macrorégionale, sans prétendre faire de l'Atlantique un espace d'intimité (Shammas, 2013). Ce n'est que dans les sciences sociales, encore une fois, qu'un espace mondial féminisé de migrations contemporaines est explicitement comparé à cet espace masculin historique qu'était l'Atlantique.

Les historiens s'accordent à reconnaître les migrations comme un facteur important de l'intégration mondiale (Manning, 2004 ; Hoerder, 2002). Notre article adopte ce point de vue et fournit une analyse sur le temps long de la répartition par genre dans les migrations internationales. Notre objectif est de contribuer à résoudre tant les problèmes de la périodisation de l'intégration mondiale que ceux soulevés par le rôle du genre dans la distinction entre un monde atlantique appartenant au passé et un présent intégré mondialement. La répartition selon le genre renvoie au nombre de femmes par rapport à celui des hommes dans toute migration ; elle est figurée par le pourcentage de personnes de sexe féminin dans les migrations ${ }^{3}$.

La répartition selon le genre, en particulier à l'échelle mondiale, est presque toujours mesurée empiriquement et analysée au moyen de méthodes quantitatives. Certaines féministes ont critiqué ces données et ces méthodes. Elles ont montré que du point de vue épistémologique, ces dernières ont participé à la construction de l'inégalité entre les sexes (Westmarland, 2001) ${ }^{4}$. Dès lors les progrès de l'analyse dans la répartition selon le genre à l'échelle mondiale tant dans les recherches sur les migrations que dans d'autres champs, sont restés limités. En 2006, un rapport de I'ONU (UNFPA, 2006) sur les migrations des femmes dans le monde a été l'une des rares études à contribuer à une " géographie du pouvoir selon les sexes " qui, selon les termes de Patricia Pessar et Sarah Mahler (2001), explore les migrations et le genre à toutes les échelles spatiales. Le présent article se réfère aux méthodes d'une science sociale positiviste tout

\footnotetext{
3 Certains chercheurs préfèrent appeler cette mesure "sex-ratio ", mais comme personne n'a jamais prétendu sérieusement que $c^{\prime}$ est le sexe biologique qui introduit des variations dans la composition des migrations, et comme on estime en général que ces variations sont provoquées par la fluidité des rapports entre les genres et les idéologies, la répartition par genre semble être le meilleur instrument d'analyse.

4 Les féministes citent souvent la formule de la poétesse Audre Géraldine Lorde (1984) :

" Les outils du maître n'abattront jamais la maison du maître ".
} 
en étant sensible aux préoccupations de la recherche féministe et en tenant particulièrement compte du genre comme phénomène idéologique et discursif.

Nous pensons que la convergence des répartitions selon le genre dans les systèmes de migration à grande échelle est un indicateur précieux de l'intégration mondiale. Ainsi, dans un monde totalement globalisé, la répartition selon le genre devrait être à peu près la même dans tous les "systèmes " de migration (Hoerder, 1999 ; Harzig et Hoerder, 2009). A contrario, dans un monde compartimenté, les répartitions vont varier dans chaque système en grande partie parce que l'idéologie du genre et les conditions matérielles de vie constituent la migration différemment en fonction des pratiques sexuées au sein de chaque système.

En mettant l'accent sur la composition de genre dans les systèmes migratoires de l'Atlantique et au-delà, apparaissent, d'un côté, une périodisation de I'intégration mondiale en tant que processus différencié sexuellement et, d'un autre côté, une perte croissante de la singularité de l'Atlantique dès le XIXe siècle. Si la convergence est bien là - d'abord pendant tout le XIXe siècle la progression de fortes proportions d'hommes dans le monde, puis un mouvement vers la parité hommes/femmes et de plus fortes proportions de femmes au début du XXe siècle -, il convient de mettre en garde contre une vision simpliste du rapport entre genre, idéologie du genre et rapports de pouvoir différenciés. Le monde n'est pas non plus devenu un espace plus intime et plus féminisé au fur et mesure qu'augmentait le nombre des femmes qui se déplaçaient. Une analyse mondiale et macrorégionale des données empiriques montre des migrations situées historiquement et géographiquement là où les rapports entre structure et discours peuvent être explorés à plusieurs niveaux.

\section{Genre et premiers systèmes migratoires modernes (1500-1888)}

La composition selon le genre des premiers systèmes migratoires concernant l'Atlantique est singulière si on la compare à d'autres systèmes de la même époque comme le système mondial de la fin du Moyen Âge, identifié par Janet Abu-Lughod (1989), où circuits commerciaux et mobilités des hommes se superposent.

Dans le monde atlantique, un système de recrutement et d'installation de la main-d'œuvre ne s'est organisé qu'après 1450, bien que d'anciens circuits afro-eurasiens persistaient. Les histoires de l'esclavage et de la construction des empires ont joué un rôle majeur dans la connaissance tant du développement du système transatlantique que des déplacements d'esclaves et de commerçants qui existaient dans I'ancien monde.

La carte Trans-Atlantic Slave Trade Database ${ }^{5}$ des premiers déplacements des esclaves africains, proposée sur le site Slave voyages, met en évidence une traite touchant plus de douze millions de personnes. Elle indique aussi qu'un

5 Voir Carte 1 : Vue d'ensemble de la traite des esclaves à partir de l'Afrique, 1500-1900, Trans-Atlantic Slave Trade Database, [en ligne] consulté le 01/08/13.

URL : http://www.slavevoyages.org/tast/assessment/intro-maps/01.jsp 
deuxième système transsaharien partant de l'Afrique centrale et occidentale pour arriver en Afrique du Nord et dans la Péninsule arabique se mettait en place alors qu'un troisième système allait de la côte swahilie, passant par la mer d'Arabie puis en Péninsule arabique jusqu'au golfe Persique, l'Asie occidentale et les côtes de I'Inde et qu'enfin un quatrième système partait de la côte swahilie et de la côte d'Afrique du sud-est pour aller dans les îles voisines - comme Madagascar - dans le sud-est de l'océan Indien. Un cinquième système, moins connu, qui n'est pas indiqué sur la carte, concernait la traite des esclaves à l'intérieur de l'Afrique subsaharienne (Manning, 1996).

Certes, les données empiriques sur les migrations forcées restent très limitées pour ce qui ne concerne pas l'Atlantique, mais les deux volumes de Women and Slavery, dirigés par Gwyn Campbell, Suzanne Miers et Joseph C. Miller (2007), offrent un bon point de départ pour une analyse comparée de la répartition hommes/femmes chez les esclaves au début de l'époque moderne. Paul Lovejoy (2007 : 259-280) indique que 75 \% des victimes de la migration transsaharienne étaient des femmes et Gwyn Campbell, Suzanne Miers et Joseph C. Miller (2007 : 4-7) estiment que près des deux tiers des esclaves vendus en Afrique orientale étaient des femmes. Inversement, sur les douze millions d'Africains soumis à la traite transatlantique, moins de $40 \%$ étaient des femmes et des jeunes filles. Cette répartition, propre à la traite transatlantique, indique clairement comment les Européens ont transformé l'esclavage en une institution qui a franchi l'Atlantique.

Dans l'Afro-Eurasie avant et après 1500, l'esclavage domestique a créé une demande de femmes esclaves pour travailler dans les maisons et s'occuper des enfants dans bien des parties du monde - par exemple chez les Vikings dans le nord de l'Atlantique, et au sein des dynasties chinoises (Seaver, 2007 : 147-148 ; Klein, 2007 : 63-82). On peut remarquer que sur cette question, les auteurs de Women and Slavery se demandent si, historiquement, la plupart des esclaves ne venaient pas d'Afrique ou n'y travaillaient pas déjà. Quoiqu'il en soit, dans l'Afrique d'après 1500, I'esclavage domestique va perdurer. Ainsi Ugo Nwokeji (2001) a montré comment certaines familles élargies africaines ont agrandi leur territoire et renforcé leur influence et leur puissance en achetant les capacités productives et reproductives des femmes esclaves. Dans la plus grande partie du monde - à l'exception, selon nombre d'auteurs, de l'Atlantique - les acheteurs préféraient les femmes parce que, dans les sociétés non organisées en États, l'esclavage servait à la fois à la famille et au lignage à des fins domestique et reproductive. Le pourcentage moins important de femmes parmi les esclaves atlantiques tenait donc très probablement à la fonction de l'esclavage comme système de main-d'œuvre adaptée aux immenses domaines de plantations créés par les investisseurs européens et les bâtisseurs d'empires. Ce modèle d'économie de plantation a gagné d'autres parties du monde amenant au XIXe siècle de nouveaux systèmes de recrutement - ce que Hugh Tinker (1974) appelle " un nouveau système d'esclavage " destiné aux plantations. Le travail salarié était également basé sur l'emploi temporaire d'hommes plutôt que sur la reproduction sur place d'une main-d'œuvre exploitée ou payée (la force de travail des asservis, assimilés à des choses, était littéralement " consommée ").

Si nous comparons les migrations transatlantiques d'esclaves à d'autres systèmes contemporains, nous remarquons combien il était rare que les 
femmes et les jeunes filles soient minoritaires parmi les esclaves migrants. Les données de la Trans-Atlantic Slave Database donnent un pourcentage d'esclaves femmes qui atteignait ou dépassait $50 \%$ (Geggus, 1989 ; Eltis et Engerman, 1992 ; Lovejoy et Richardson, 1995). Ainsi les travaux de Jennifer Morgan (2004) sur l'esclavage dans les Antilles britanniques et l'Amérique du Nord coloniale soulignent que les acheteurs d'esclaves, particulièrement durant les deux premiers siècles de ce commerce, se souciaient autant de la reproduction de leur main-d'œuvre forcée (en achetant des femmes) que de l'acquisition de travailleurs. Les dimensions domestiques et reproductrices d'anciens systèmes n'étaient apparemment pas totalement ou automatiquement dissipées par l'air salé de I'Atlantique. Les archives mettent en évidence la préférence des acheteurs européens pour des esclaves hommes, mais les comparaisons entre des régions envoyant des cargaisons d'esclaves des deux sexes - un peu plus de femmes venant du golfe du Biafra, par exemple, et un peu moins venant d'Afrique centrale occidentale - indiquent aussi comment l'état des rapports entre les sexes en Afrique (conséquence des guerres, trafic d'esclaves, etc.) pouvait contraindre les choix des Européens en intervenant sur la reproduction des captifs masculins à vendre (Miller, 1988 ; Nwokeji, 2010). Lorsque la répartition hommes/femmes chez les esclaves tendait à s'équilibrer, comme en Amérique du Nord, la main-d'œuvre se reproduisait et des espaces d'intimité ainsi que des familles d'esclaves se formaient sur les terres des plantations où elles étaient exploitées (Gutman, 1976).

Les esclaves africains des Amériques présentaient une autre particularité. Les études sur la traite transatlantique ont souligné qu'il y avait parmi eux une proportion de femmes relativement plus élevée par rapport à presque toutes les migrations d'origine européenne de l'Atlantique à la même époque (Eltis, 1983). Entre 1500 et 1800, trois à quatre millions d'Européens (dont 500000 à un million et demi venant d'Espagne ; un et demi du Portugal, pourtant moins peuplé ; 400000 de Grande Bretagne ; 100000 d'Allemagne ; 75000 de France) traversèrent I'Atlantique. Environ $80 \%$ de ces Européens étaient en quelque sorte sous contrat ou obéissaient aux " ordres " de leurs supérieurs (militaires de carrière, missionnaires catholiques). Les $20 \%$ restant étaient constitués de réfugiés politiques ou de religieux, d'artisans, d'aventuriers, d'hommes d'affaires, d'administrateurs coloniaux et enfin d'investisseurs (O'Reilly, 2012). En outre, un nombre indéterminé d'Européens ont également rejoint les colonies européennes dans le Pacifique et un nombre important d'entre eux s'est déplacé à l'intérieur de l'Europe (Lucassen et Lucassen, 2009).

La proportion d'hommes dans les flux transatlantiques au début de l'époque moderne était beaucoup plus élevée chez les migrants les plus démunis que chez les esclaves africains. Bien entendu, les soldats et les missionnaires étaient presque tous des hommes, tandis que chez les " domestiques " espagnols au XVle siècle, le pourcentage de femmes passe de 5,6 - 6,3\% au début du siècle à 28,5\% dans les années 1560 et 1570 pour retomber ensuite (Boyd-Bowman, 1976a). Nous retrouvons le même déséquilibre dans la répartition des domestiques sous contrat de l'Empire britannique. Les premiers domestiques envoyés en Virginie ne comportaient que $11 \%$ de femmes (Moller, 1945) et un registre tenu en 1635 par des douaniers anglais mentionne $6 \%$ de femmes parmi les migrants partant aux Antilles (Games, 1999). Au XVIIle siècle, la proportion de femmes dépassait rarement $25 \%$ des migrants quittant l'Europe pour 
l'Amérique du Nord (Bailyn, 1986b). On a souvent avancé que, contrairement à ce que nous pensons aujourd'hui, nombre de ces migrants - issus d'une tradition européenne où l'on quittait sa famille à l'adolescence pour se faire embaucher ou entrer en apprentissage - espéraient peut-être que leur séjour aux Amériques serait bref ${ }^{6}$.

En revanche, les groupes composés de migrants légèrement plus prospères - colons espagnols ou britanniques venus pour s'installer - étaient moins nombreux et comptaient une proportion beaucoup plus élevée de femmes et de jeunes filles. Peter Boyd-Bowman a même conclu ses recherches sur les migrants quittant l'Espagne au XVle siècle par deux portraits contrastés. Le premier évoque I'homme sans ressource : "Andalou appauvri [...] vingtsept ans et demi, célibataire, non qualifié et probablement à demi illettré, poussé par la faim à partir au Pérou au service de qui lui paierait le voyage et lui procurerait les autorisations nécessaires "; le second décrit une femme qui migre pour s'installer : " un peu plus de trente ans [...] partant au Pérou avec un mari de trente-six ans, deux jeunes enfants, un domestique, et une bonne " (1976: 732).

Les réfugiés, les puritains et les divers dissidents religieux qui partaient pour l'Amérique du Nord britannique voyageaient souvent en famille ; les femmes comptaient pour un tiers (Games, 1999). Dans ce qui a été appelé la grande migration puritaine vers le Massachusetts (1630-1640), Dejohn a relevé jusqu'à $40 \%$ de femmes, et au moins un navire transportant une majorité de femmes (1985). Enfin, Bernard Baylin (1986b), dans son tableau des Écossais recrutés comme colons par des Américains du nord qui spéculaient sur des terrains, décrit des familles équilibrées qui exploitaient des fermes.

Pour ce qui est des autres migrants quittant l'Europe au début de l'époque moderne, ils ressemblaient davantage à la main-d'œuvre sous contrat qu'aux colons. L'Angleterre a ainsi expédié nombre de pauvres condamnés, dans les colonies pénitentiaires du Pacifique nouvellement créées pendant la seconde moitié du XVIIle siècle (Bullard, 2000 ; Ekirch, 1987 ; Grubb, 2000 ; Morgan et Rushton, 2004). Des recherches menées sur l'Australie et la Nouvelle-Zélande montrent que les femmes représentaient 18 à $20 \%$ des condamnés (Robson, 1994). Les historiens qui ont récemment analysé la situation des migrants en Europe entre 1500 et 1800 estiment leur nombre à 107,9 millions, soit bien plus que I'ensemble des voyageurs transatlantiques quittant l'Europe et l'Afrique; ils les décrivent aussi comme occupant des professions majoritairement masculines telles que celles rattachées aux corps des militaires, des apprentis et des marins. Dans les groupes étudiés par Jan Lucassen et Leo Lucassen (2009), seuls peut-être les vagabonds comptaient un nombre important de femmes et d'enfants. En tout cas, à cette époque, il est possible que la proportion de femmes n'ait pas été plus élevée dans les déplacements locaux que dans les voyages sur de longue distance.

Dans l'ensemble, les systèmes de migration transatlantiques se caractérisaient par de plus faibles pourcentages de femmes que dans les systèmes de migration d'esclaves en provenance d'Afrique et d'Eurasie. L'examen de

6 Pour les Espagnols, voir Altman (1989); pour les Britanniques, voir Matt (2011). 
nouvelles données sur l'Atlantique laisse voir une convergence dans la composition par genre chez les esclaves africains et les Européens sous contrat. La sociologue Katharine Donato et I'historienne Donna Gabaccia ont précisé qu'au cours du XVIIle siècle la répartition par genre des esclaves africains s'est rapprochée de celle des migrants européens sous contrat, tandis que le nombre d'esclaves n'augmentait plus et que la proportion de femmes diminuait (Gabaccia et Donato, 2015). À la fin du XVIIle siècle, le système transatlantique d'esclavage, le système britannique, moins important, de prisonniers européens bannis ainsi que le système atlantique de travailleurs forcés sous contrat, étaient devenus nettement plus masculins, la proportion de femmes variant de $20 \%$ (chez les Européens) à $35 \%$ (chez les Africains). Nous ne pouvons aujourd'hui qu'émettre l'hypothèse que d'autres systèmes de migrations afro-eurasiens - un petit nombre de commerçants et marins, peut-être - ressemblaient davantage aux travailleurs forcés de l'Atlantique qu'aux esclaves d'Afrique et d'Eurasie. Néanmoins, les systèmes transatlantiques se distinguaient dans un monde où les migrations de masse étaient en grande partie composées d'esclaves, dont la plupart (I'Atlantique excepté) étaient des femmes.

\section{Genre et migrations au XIXe siècle}

Dans ce qu'a défini I'historien Eric Hobsbawn comme le "long XIXe siècle " (de 1800 à l'après Grande Guerre), I'abolition de I'esclavage transatlantique et l'expansion du mode de production des plantations dans les empires européens qui s'étendaient en Afrique et en Asie ont entraîné un changement dans la qualification des travailleurs européens salariés : de jeunes apprentis et de domestiques sous contrat à ouvriers d'usine et employés sous contrat pour les plantations. Le XIXe siècle allait devenir, pour certains démographes, l'époque des " migrations prolétaires de masse " (Wilcox et Ferenczi éds, 1970). La plupart de ces prolétaires étaient des hommes.

Dans une large mesure, le XIXe siècle voit s'opposer deux modèles : une migration transatlantique constituée d'une main-d'œuvre largement masculine souvent contrainte et une migration de colons européens bâtisseurs d'empires où la répartition par genre était plus équilibrée. Les salariés européens et asiatiques finissent par devenir beaucoup plus nombreux que les colons européens. Déjà au début du XIXe siècle, le système transatlantique était devenu moins spécifique, et, à la fin du siècle, les migrations transatlantiques à prédominance européenne masculine étaient devenues la norme dans le monde. Si nous nous intéressons à la répartition par genre comme critère d'intégration mondiale de l'Atlantique, il faut bien reconnaître que celle-ci était presque achevée pendant la seconde grande période de mondialisation du XIXe siècle (Williamson, 1996 ; O'Rourke et Williamson, 1999 ; Robertson, 2003). Cette conclusion est confirmée par la féminisation des migrations mondiales qui commençaient au XXe siècle.

Dans une première tentative de réunir et d'analyser les données sur les migrations à l'échelle mondiale, Adam McKeown (2004) a estimé que le total des migrants transcontinentaux, entre 1846 et 1940, s'établissait entre 155 et 172 millions et demi. Selon I'auteur, cinquante-cinq millions de personnes ont quitté l'Europe et le Moyen-Orient et environ trois millions ont quitté I'Inde et l'est de l'Asie pour les Amériques, cinquante autres millions sont partis de 
I'Asie du sud et de Chine du sud, cinq millions du Moyen-Orient et d'autres parties de la Chine en direction de I'Asie du sud-est, de I'Australie et d'autres destinations dans le bassin du Pacifique. Pas moins de quarante-huit millions de personnes ont quitté I'Asie orientale pour des régions frontalières en Asie centrale, en Sibérie et en Mandchourie. McKeown ne donne pas d'estimation des déplacements internationaux à l'intérieur de l'Europe (là où il n'y avait pas de frontière évidente plus proche que la Sibérie ou I'Ukraine), mais le nombre de migrants internationaux et intra-européens a certainement atteint, s'il ne l'a pas largement dépassé, celui des cinquante-cinq millions d'Européens partis pour les Amériques selon les estimations de Harzig et Hoerder (2009 : 36).

Si l'on combine les chiffres donnés par McKeown et les analyses macrorégionales des migrations présentées dans l'ouvrage Connecting Seas and Connected Ocean Rims (Gabaccia et Hoerder éds, 2011), se dessine alors une carte du monde comprenant huit grands systèmes migratoires au XIXe siècle. Au nord et au sud de l'Atlantique, nous trouvons des migrations venant de différentes parties de l'Europe et un système transpacifique avec des Asiatiques qui se dirigeaient vers l'Atlantique, les Caraïbes, et les deux Amériques. II faut aussi ajouter le vieux système intra-européen qui attirait les travailleurs des périphéries de l'Europe vers les centres industriels et les débuts du système impérial britannique de diaspora qui continuait à envoyer des migrants britanniques et irlandais vers d'anciennes colonies en Amérique du Nord (Canada, États-Unis), aux antipodes (Australie et Nouvelle-Zélande) et en Afrique du Sud. Sur les côtes d'Asie et d'Afrique, se déployait un système dans les mers au sud de la Chine reliant l'est de I'Asie au Sud-Est avec les colonies minières et les plantations. Le système de I'océan Indien mettait en relation les principales colonies britanniques en Inde, I'arrière-pays de Hong Kong, et l'Afrique. Il existait enfin un système sibérien-mandchourien qui incluait des populations arrivant de l'est et de l'ouest de l'Asie, de l'Europe de l'Est et même de l'Europe centrale.

Dès la première moitié du XIXe siècle, les conséquences de I'abolition progressive de l'esclavage transatlantique sur la composition par genre des migrants sont attestées bien au-delà de l'Atlantique, grâce en grande partie à l'expansion de l'Empire britannique en Asie et dans le sud du Pacifique (Gabaccia et Hoerder éds, 2011). En s'appuyant sur les données de I'Atlantic Slave Trade Database concernant les flux de migration, nous pouvons observer une synchronisation croissante dans la répartition par genre pendant la période de I'abolition. Le tableau 1 montre les variations par sexe dans la traite transatlantique, I'immigration transatlantique en Amérique du Nord (avant 1835) et l'immigration impériale et pénitentiaire en Australie et en Nouvelle-Zélande. Cependant sur I'Atlantique, I'existence de l'esclavage compliquait tellement le servage européen que les États-Unis, depuis peu indépendants, avaient arrêté les migrations sous contrat en 1830 (Steinfeld, 1991 ; Grubb, 1994 ; Eltis, 1983). Cette mesure a mis fin au synchronisme dans la composition par genre entre les migrants européens et les esclaves qui arrivaient. L'abolition de l'esclavage dans I'Atlantique a continué à avoir des conséquences dans le Pacifique où le synchronisme des répartitions entre esclaves et travailleurs sous contrat persista au moins jusqu'en 1860. L'envoi et l'installation de condamnés en Australie se prolongèrent également jusqu'à cette date. 
Graphique 1 : Pourcentage de femmes parmi les esclaves africains et les immigrants entrant en Amérique du Nord, en Australie et en Nouvelle-Zélande, 1800-1860

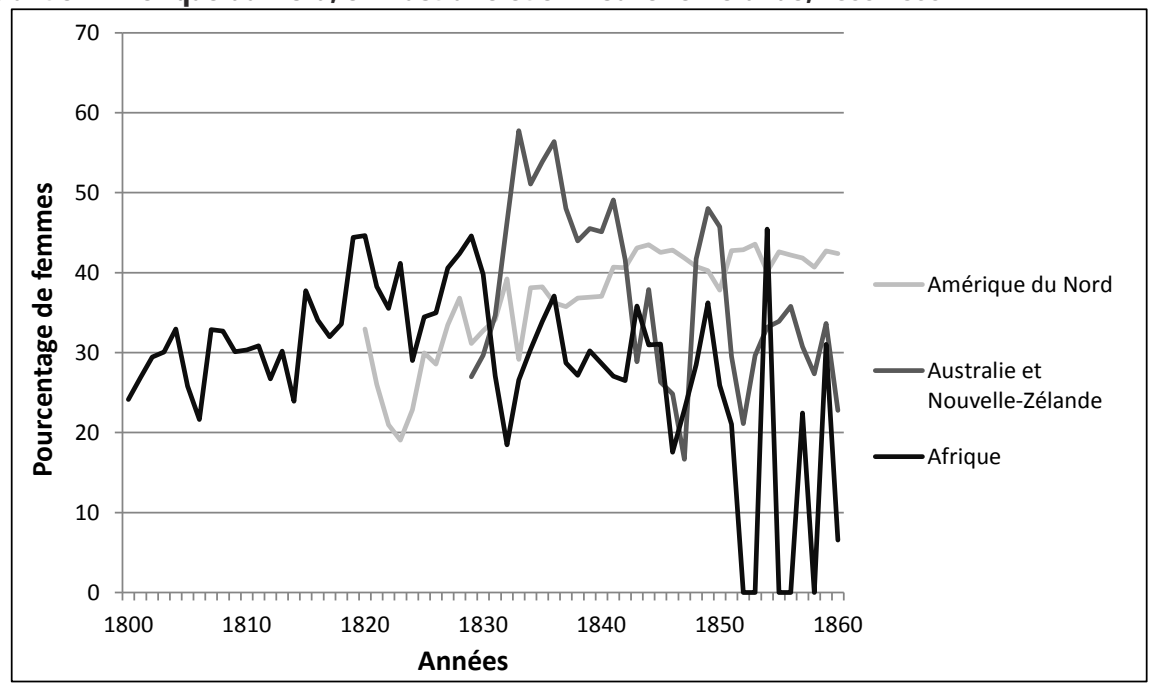

Source : Voyages: The Trans-Atlantic Slave Trade Database, [en ligne] consulté le 01/08/13. URL: http://www.slavevoyages.org/tast/database/download.faces. Walter Willcox et Imre Ferenczi (1970).

Alors que l'augmentation du pourcentage de femmes et de jeunes filles accompagnait le déclin des migrations européennes pénitentiaires et sous contrat vers I'Amérique du Nord, I'Australie et la Nouvelle-Zélande, il en allait différemment des colonisations de peuplement dans ces deux parties du monde. La proportion de femmes chez les Européens entrant aux États-Unis augmenta régulièrement de 1820 à 1840 puis se stabilisa à un peu plus de $40 \%$, témoignant que ces groupes de colons partaient vers l'ouest en famille, pour s'y installer en tant que sujets libres, blancs, et futurs citoyens (Foner, 1995). Au contraire, plus au sud, l'équilibre entre les sexes, avec parfois une majorité féminine, devint la norme après 1830. Cela découlait de la planification impériale, les stratèges britanniques espérant que des femmes célibataires amélioreraient la moralité et les chiffres des mariages en Australie, rendant plus dociles les populations de Botany Bay où les hommes étaient en forte majorité. L'assistance financière de la couronne, en particulier pour les jeunes couples mariés et les " cargaisons " d'hommes et de femmes célibataires en proportions égales, eut pour effet que les proportions de femmes étaient plus élevées que chez ceux qui partaient s'installer en Amérique (Haines, 1994 ; McDonald et Richards, 1997 ; Hammerton, 1979).

La crise économique de 1841 ayant brutalement mis fin aux systèmes d'aide à l'émigration vers l'Australie, la composition selon le genre dans cette migration se différencia brutalement de celle de l'Amérique du Nord. C'était là l'annonce de la masculinisation qui allait bientôt caractériser presque toutes les migrations de main-d'œuvre dans le monde. En Australie, la remontée du pourcentage d'hommes accompagna la découverte d'or en 1851 (Graphique 1) : ce pour- 
centage à la fin des années 1840 et dans la décennie suivante atteignait parfois la très forte proportion d'hommes enregistrée dans les migrations forcées de main-d'œuvre du siècle précédent.

À la fin du XIXe siècle, la masculinisation était la norme dans presque tous les systèmes de migration. Bien que nous disposons de peu de données empiriques sur les travailleurs quittant I'Asie au XIXe siècle, la plupart des recherches montrent que ceux qui partaient de Chine et d'Inde, libres ou sous contrat, étaient à très forte majorité des hommes (Lee, 1989 ; Jain et Reddock éds.,1998 ; Sen, 2004). Mais ces deux migrations ont connu une évolution différente et il en a découlé une distinction dans les répartitions par genre (Northrup, 1995). Les administrateurs de l'Empire britannique, avec leur longue expérience des déportations pénitentiaires de l'Angleterre vers l'Australie et d'Inde vers l'Île Maurice, considéraient que la régulation de l'équilibre entre les sexes était indispensable au maintien de l'ordre colonial (Anderson, 2000). Ils veillèrent à ce que des proportions fixes de femmes (de $15 \%$ à $40 \%$, selon les dates et les lieux) accompagnent les travailleurs sous contrat (Northrup, 1995). En 1870, des travailleurs chinois et indiens étaient sous contrat dans divers endroits comme le Panama, le Pérou, la Malaisie, I'Australie, Trinidad, la Guinée britannique, la Jamaïque, et le Honduras britannique (Campbell, 1923 ; Stewart, 1951 ; Cohen, 1971 ; Meagher, 2003). Mais aux Antilles anglaises, il n'y avait que $14 \%$ de femmes et leur proportion chez les Chinois était encore plus faible, accentuant le contraste avec les travailleurs indiens et provoquant des attaques racistes et nationalistes contre les Chinois accusés d'homosexualité et aussi de constituer une menace sexuelle pour les femmes indigènes (Samaroo, 2013 ; Lai, 1993 ; Lopez, 2013). Même chez les Chinois sans contrat - la majorité de ceux qui cherchaient du travail en Amérique du Nord - les hommes l'emportaient largement.

Puisque les principales sources de données sur les migrations au XIXe siècle étaient produites par des États-nations (ou parfois des colonies semi-autonomes), les témoignages concernant cette époque ne coïncident pas exactement avec les huit grands systèmes décrits (voir supra). Mais si nous regardons les chiffres concernant la répartition par genre chez les émigrants et les immigrants sur la durée, on prend la mesure de la masculinisation et de la persistance de variations d'un système à l'autre. Le graphique 2 compare les émigrants quittant I'Europe et l'Asie selon les chiffres réunis par le Bureau international du travail dans les années 1920 (Wilcox et Ferenczi éds., 1970), chiffres complétés par ceux concernant les émigrants chinois ${ }^{7}$. Le graphique 3 analyse les données relatives aux personnes entrant aux États-Unis, en Amérique du Sud, en Australie et en Nouvelle-Zélande, ainsi qu'en Europe du nord-ouest pendant la même période.

Considérés ensemble, ces tableaux confirment que les migrations dans le Pacifique, pendant presque tout le XIXe siècle, restèrent bien plus largement masculines que celles arrivées aux États-Unis et en Amérique du Sud, principalement transatlantiques. Le graphique 2 conforte encore la forte prédominance masculine dans les arrivées depuis la Chine (McKeown, 2001). Dans le système de I'Atlantique nord, les États-Unis attiraient bien plus de femmes que I'Amérique du Sud, peut-être parce que davantage d'immigrants aux États-Unis

7 L'historienne Sinn a compilé des données issues des archives de Hong Kong et celles des ports sur le Pacifique (2013). 
Graphique 2 : Pourcentage de femmes parmi les immigrants en provenance de l'Europe du Nord-Ouest, de l'Europe du Sud-Est, du Japon et de la Chine entrant en Amérique, 1860-1924

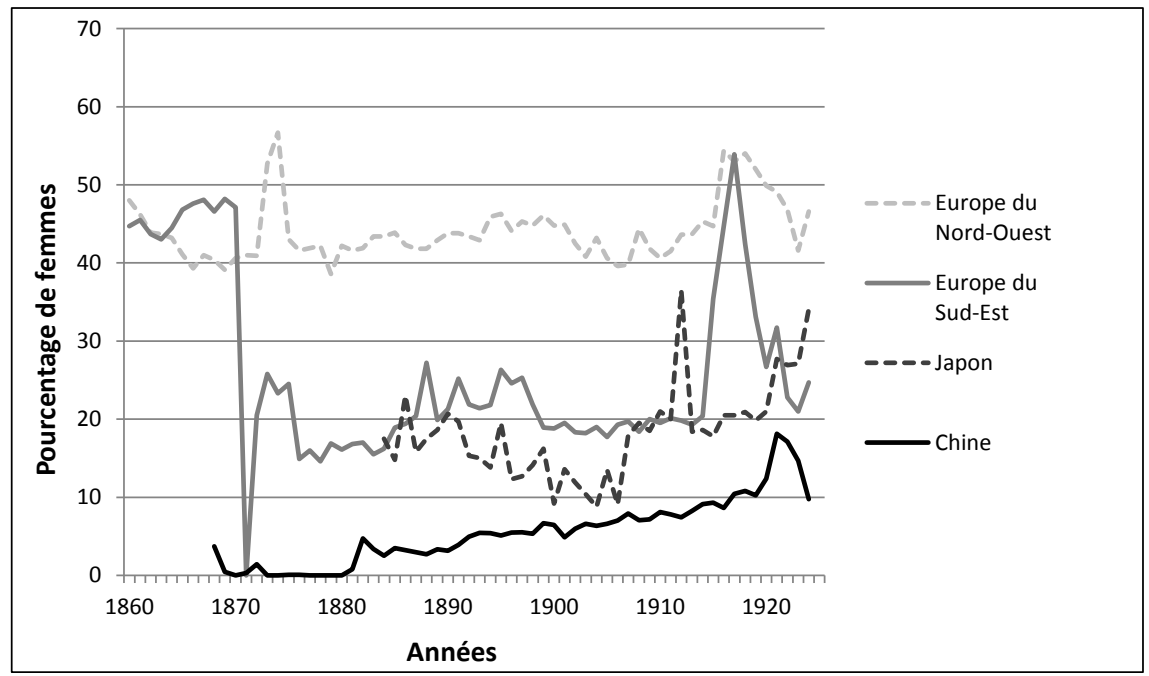

Source : Walter Willcox et Imre Ferenczi (1970). Chiffres sur la migration chinoise aimablement fournis par Adam McKeown et Elizabeth Yuk Yee Sinn.

Graphique 3 : Pourcentage de femmes parmi les immigrants entrant aux États-Unis, en Amérique du Sud, en Australie et Nouvelle-Zélande et en Europe du Nord-Ouest, 1860-1924

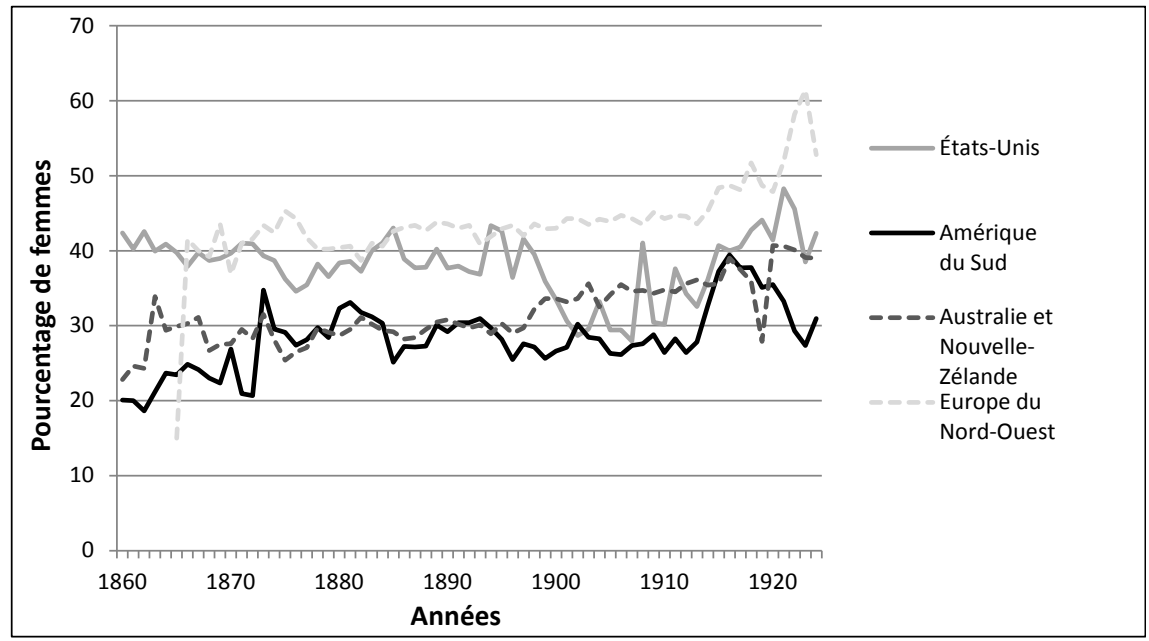

Source : Walter Willcox et Imre Ferenczi (1970). 
venaient d'Europe occidentale et septentrionale, et que ceux-ci comprenaient une proportion de femmes nettement plus élevée que chez les Européens du sud et de l'est. La chute brutale du pourcentage de femmes dans les entrées aux États-Unis au cours des dernières décennies du XIXe siècle reflète presque certainement un passage entre ceux qui venaient de l'Europe du nord et de l'ouest et ceux qui venaient du sud et de l'est - ceci s'appliquant à un moindre degré à l'Asie et au Mexique (Gabaccia, 1996). À la fin du XIXe siècle la composition par genre dans le système de l'Atlantique nord est retombée au niveau de I'Atlantique sud qui attirait surtout les migrants de l'Europe du sud. Elle converge au sein de deux systèmes ainsi qu'avec le système de migration du Pacifique, alors que le pourcentage de femmes au départ d'Asie augmentait lentement. Le système de migrations intra-européennes se distinguait nettement : il avait atteint le plus fort pourcentage de femmes, en raison, probablement, de la demande d'employées de maison dans les villes en expansion de l'Europe du nord. L'historien Marlou Schrover (2013) a soutenu que l'équilibre avait été atteint chez les émigrants européens au XIXe siècle, bien avant les autres systèmes migratoires dans le monde.

Le pourcentage de femmes à l'échelle mondiale était quasiment tombé à $25 \%$ ou moins vers la fin du XIXe siècle, un peu plus bas qu'en Atlantique au XVIIle siècle. Des études de cas portant sur les systèmes de l'Atlantique et du Pacifique ont souvent apporté la même explication à la très forte proportion d'hommes à la fin du XVIIle siècle. Les hommes qui quittaient l'Europe du sud-est et la Chine ne trouvaient, en général, que du travail temporaire ou saisonnier dans l'agriculture, les mines et le bâtiment; la rotation était très forte et ils retournaient rapidement dans leur patrie, où de nouvelles générations de travailleurs migrants naissaient et grandissaient (Gabaccia, 1997, 2000 ; McKeown, 2001). Avant 1880, Indiens et Chinois travaillaient sur les plantations coloniales à Cuba ou au Brésil (rarement les Européens), souvent aux côtés des esclaves. Les populations quittant l'Europe et l'Asie étaient composées d'hommes mariés ou de célibataires qui voyageaient sans leur famille mais en groupes d'amis et de parents, parfois dirigés ou contrôlés par un recruteur de main-d'œuvre appelé "boss " ou " passeur " (Gabaccia, 1997). Plus ces migrants étaient itinérants et plus ils étaient majoritairement des hommes en âge de travailler, allant de 15 à 55 ans. Les études sur les Chinois et les Italiens soulignent la motivation de ces hommes à envoyer de l'argent à leur famille, quand bien même des " campagnes " de travail salarié, plus ou moins longues, donnaient lieu à la constitution de ménages qui travaillaient dans l'agriculture de subsistance (Ramirez, 1991). Les inégalités structurelles dans un marché du travail de plus en plus mondialisé créaient quelques ressemblances entre les migrants travailleurs libres qui arrivaient d'Europe et les travailleurs asiatiques qui voyageaient sous contrat, et qui rencontraient des restrictions et des obstacles de plus en plus forts dans leurs déplacements (McKeown, 2008).

Dans les tableaux 2 et 3, nous observons qu'autour de 1900 se dessine un renversement de ces tendances annonçant l'apparition d'un phénomène de féminisation variable d'un système à un autre. Dans certains cas, cette tendance s'est inversée notamment durant la Grande Guerre. Dans le système intra-européen au cours des années 1920, les femmes étaient plus nombreuses que les hommes, tandis qu'aux États-Unis et dans le Pacifique sud, le pourcentage de femmes dépassa rapidement $40 \%$, puis cessa d'augmenter. Certes la participation des 
émigrantes chinoises restait peu élevée, mais Adam McKeown a comparé les données concernant des Chinois et des Européens partant et rentrant et a noté le même processus de féminisation au XXe siècle mis en évidence dans les graphiques 2 et 3 . II en déduit une convergence mondiale de la représentation féminine à cette époque (McKeown, 2010). Ces études de cas, qui ont montré la masculinisation des populations émigrant au XIXe siècle, n'ont cependant pas expliqué les données des graphiques 2 et 3 , à savoir les migrations à dominante féminine avec une répartition équilibrée au début du XXe siècle.

\section{Féminisation au XXe siècle et fin de la singularité de I'Atlantique}

Tout au long du XXe siècle, la faible féminisation (Graphiques 2 et 3) s'est non seulement maintenue mais elle est devenue de plus en plus visible et consistante dans tous les systèmes migratoires. La singularité de l'Atlantique est devenue de moins en moins perceptible, comme Hania Zlotnik l'a fait remarquer avec bon sens à I'ONU :

"Dès 1960, les émigrantes ont représenté presque 47 \% de ceux qui vivaient loin de leur pays de naissance. Depuis, cette proportion augmente régulièrement dans le monde entier, atteignant $48 \%$ en 1990 et presque $49 \%$ en 2000. Certes, cette tendance accom-

pagne une "féminisation " accrue dans les migrations internationales, mais pareille augmentation est faible comparée au haut niveau de féminisation existant déjà en 1960 "

(Zlotnik, 2003).

Les données de Zlotnik n'étaient pas regroupées selon les systèmes migratoires mais - à l'image des recensements d'où elles étaient issues - elles révélaient qu'en 2000 l'Afrique du nord et l'Asie du sud avaient le plus faible pourcentage de femmes (respectivement $12,8 \%$ et $44,4 \%$ ) parmi leurs immigrants résidents. Par rapport aux variations importantes au début de l'époque moderne dans les systèmes de l'Atlantique et autres, les variations entre les régions où le pourcentage de femmes dépassait la moyenne en 2000 (52,4 \% en Europe, 51 \% en Amérique du Nord, 50,5 \% en Océanie et en Amérique du Sud) paraissent modestes. II s'agit d'un autre indicateur de convergence mondiale. Les remarques de Zlotnik ont lancé un vaste défi - resté sans réponse - aux nombreux chercheurs en sciences sociales qui, au début des années 1990, ont présenté la féminisation des migrants comme une nouvelle dimension de la mondialisation de la fin du XXe siècle en I'opposant à la dominante masculine dans I'Atlantique au XIXe siècle (Castles et Miller, 2009). En fait, au XIXe siècle, la suprématie masculine était mondiale et elle ne constituait pas un phénomène réservé à l'Atlantique. C'est le glissement vers un équilibre entre les sexes au niveau mondial qui réclame une interprétation.

Trois explications à la féminisation des migrations mondiales, lesquelles sont différentes mais compatibles, sont couramment avancées. Le premier facteur concerne, les restrictions imposées partout aux immigrations. Selon Gabaccia et Donato (2015), le XXe siècle a vu le début et la généralisation de mesures visant à limiter les libres déplacements de travailleurs hommes non qualifiés cherchant du travail entre 1850 et 1914. Au milieu du XXe siècle, I'hostilité et le rejet ne visaient plus seulement les travailleurs asiatiques, ils s'appliquaient 
aussi à ceux qui venaient de I'Europe du Sud et de I'Est. En outre, contrairement à une croyance largement répandue, les migrations de travailleurs et de réfugiés sont plus faibles aujourd'hui, par rapport aux chiffres de la population mondiale, que celles du XIXe siècle. Et, à mesure que le nombre d'immigrants hommes diminuait, le pourcentage de femmes migrantes au niveau mondial s'élevait. Pour Katherine Donato et ses collègues le second facteur relève du vieillissement d'anciennes cohortes d'immigrants qui a contribué à l'augmentation du pourcentage de femmes, en particulier dans les régions où l'immigration avait une longue histoire comme les Amériques du Nord et du Sud, l'Europe et l'Océanie (Donato et al., 2011). Enfin, dans leur récent volume collectif Proletarian and Gendered Mass Migrations: A Global Perspective on Continuities and Discontinuities from the 19th to the 21st Centuries, publie en 2013, les historiens Dirk Hoerder et Amarjit Kaur présentent une série d'études de cas qui font de l'augmentation mondiale de la demande de travailleuses dans le care et les industries de la santé le troisième facteur de féminisation à l'échelle mondiale. Ces analyses montrent que les raisons de la féminisation des migrations mondiales sont très proches de celles qui expliquent l'équilibre des migrations intra-européennes au XIXe siècle.

\section{Données empiriques dans les études atlantiques et les études de genre}

Cet article a présenté une série de convergences dans la répartition selon le genre dans les migrations qui suggère une intégration ancienne de l'Atlantique dans les marchés mondiaux de la main-d'œuvre masculine et féminine. Ce n'est certes qu'un des indicateurs d'intégration - à considérer en tenant compte du commerce, des relations internationales et de la circulation des idées -, mais il constitue un apport important à une histoire de I'Atlantique sur le temps long. Si le XIXe siècle a vu l'intégration presque complète de l'Atlantique dans les marchés mondiaux pour la main-d'œuvre masculine en déplacement, il est tentant de voir la transition vers la féminisation et l'arrivée globale des restrictions des migrations dans les années 1920 et 1930 comme sonnant le glas de cet "Atlantique sur le temps long " distinct.

La convergence mondiale vers des migrations équilibrées par genre a accompagné une nouvelle géographie des migrations organisée non pas autour de systèmes transocéaniques est-ouest mais de systèmes hémisphériques nord-sud. II est frappant de constater que cette nouvelle géographie n'a pas été définie comme masculine ou féminine comme l'ont été par exemple les déplacements sur de courtes et de longues distances au XIXe siècle. Hoerder et Kaur (2013) consacrent une section à l'Atlantique dans leur récent volume collectif d'études de cas sur la migration des travailleurs du point de vue du genre, mais il est clair que leur Atlantique est déjà composé au XIXe siècle de systèmes continentaux distincts. Les auteurs examinent les déplacements intra-européens et ils étudient séparément le changement dans l'immigration en Amérique du Nord, atlantique puis pacifique et latino-américaine, les systèmes intérieurs 
à l'Amérique du Sud et à la Russie-URSS ${ }^{8}$. Dans tous ces systèmes, les femmes migrantes sont décrites comme des agents historiques jouant un rôle capital dans toutes les migrations, même celles à forte prédominance masculine.

L'examen attentif de données empiriques sur la composition par genre au long d'un demi-millénaire dans I'histoire des migrations et à travers des macrorégions toujours en mutation montre tout ce qui reste à faire au-delà de la périodisation de l'intégration mondiale de I'histoire de l'Atlantique. II laisse également entrevoir de nouveaux domaines de discussion entre les spécialistes des Women Studies et les chercheurs travaillant avec les données et les méthodes des sciences sociales. Notre analyse devrait rappeler au lecteur que, tout d'abord, la féminisation des migrations s'explique peut-être par la grande diversité des idéologies sur le genre et les relations de pouvoir. La vente de membres d'une famille jugés superflus a pu engendrer des migrations d'esclaves dans I'Afrique occidentale aux débuts de l'époque moderne. Mais c'était aussi le cas de politiques impériales au XIXe siècle qui voyaient les femmes comme celles qui pouvaient " apprivoiser " les célibataires " hors la loi " dans les Nouvelles-Galles-du-Sud, ou lorsque de jeunes femmes - Irlandaises au XIXe siècle ou Mexicaines aujourd'hui - pensaient qu'aller s'installer dans une grande ville de l'autre côté de la frontière pour travailler comme domestique était une chance. En définitive, il est hautement improbable que l'on trouve une unique explication à la féminisation ou la masculinisation d'un groupe.

II est clair également que la composition par genre ne régissait pas les rapports de pouvoir chez les émigrants. Si les migrations d'esclaves afro-eurasiennes étaient féminisées, cette féminisation n'était pas un effet de la responsabilisation des femmes, pas plus que cette responsabilisation n'était un effet de leur esclavage. Dans l'ancien monde, l'esclavage était surtout à l'œuvre dans des espaces privés et domestiques ; même au niveau local, l'intimité était bien plus difficile à atteindre pour les esclaves ou même pour les domestiques sous contrat. Au contraire, dans l'Atlantique, dans les systèmes européens impériaux, et dans le système intra-européen, l'entrelacement complexe du travail féminin et de la reproduction - caractéristique de l'esclavage traditionnel dans les sociétés précapitalistes et non-étatiques - avait déjà été isolé. Quand les populations d'esclaves ont commencé à se reproduire en Amérique du Nord, il en a découlé non pas un accroissement de l'intimité ou du pouvoir féminin mais la fragmentation par la force de foyers et de familles d'esclaves pour fournir de nouvelles générations de travailleurs aux plantations de coton dans I'économie florissante d'avant la guerre de Sécession. De rares recherches théoriques et empiriques, laissent voir comment le déséquilibre entre les sexes peut avantager le plus faible dans ses rapports personnels ou conjugaux avec le plus fort, sans pourtant avancer que l'exercice du pouvoir entre personnes affaiblit les inégalités entre les sexes exprimées par des idéologies ou des différences structurelles (Guttentag et Secord, 1983).

L'essentiel est pourtant que les témoignages empiriques n'affaiblissent nullement l'effort pour créer une relation stable entre le genre et l'échelle

8 Le reste du volume est également organisé autour de systèmes sous-continentaux - les Asie, les Afrique -, seule la Méditerranée subsiste comme canal menant à I'Atlantique pour les Syriens du Liban et en Europe pour les Africains du Nord. 
spatiale des mobilités ; les chercheurs sont donc contraints de se poser de nouvelles questions sur la manière et les conditions dans lesquelles le genre intervient dans le discours sur l'espace créé par les systèmes de migration. À l'exception peut-être des migrations du XIXe et du XXe siècles à l'intérieur de l'Europe, toutes les migrations - équilibrées ou à dominante féminine examinées dans cet article se sont déroulées sur de très longues distances. L'augmentation du nombre de femmes et de jeunes filles traversant l'Atlantique et le Pacifique n'a pas automatiquement rendu plus féminine, plus intime, plus domestique ni la mobilité physique ni la construction discursive des espaces traversés. Que la prédominance des femmes ait (ou non) fait de l'esclavage d'Afrique et d'Eurasie une institution au discours plus féminin à côté de l'esclavage " masculin " de l'Atlantique, voilà qui mérite discussion étant donné le rôle essentiel de la féminisation du Moyen-Orient par l'orientalisme occidental. Certes, la féminisation des migrations bien avant 1960 n'a presque pas eu d'effet sur la représentation de l'émigrant, qui est resté un sujet masculin, dans les sciences sociales (Morokvasic, 1984), mais l'importance aujourd'hui du travail du care et du commerce du sexe, dans les discours de "panique morale ", pourrait finir par faire de la victimisation des femmes un symbole de la mondialisation contemporaine - au même titre que la figure menaçante et primitive de I'homme qui migrait pour chercher du travail a symbolisé l'économie mondiale du XIXe siècle.

\section{Références bibliographiques}

Abu-Lughod Janet (1989) Before European Hegemony: The World System A.D.1250-1350, Oxford, Oxford University Press, 443 p.

Altman Ida (1989) Emigrants and Society: Extremadura and America in the Sixteenth Century, Berkeley, University of California Press, 372 p.

Anderson Clare (2000) Convicts in the Indian Ocean: Transportation from South Asia to Mauritius, 1815- 1853, New York, St. Martin's Press, 192 p.

Bailyn Bernard (1986a) The Peopling of British North America: An Introduction, New York, Alfred A. Knopf, 192 p.

Bailyn Bernard (1986b) Voyagers to the West: A Passage in the Peopling of America on the Eve of the Revolution, New York, Alfred A. Knopf, 668 p.

Boyd-Bowman Peter (1976a) Patterns of Spanish Emigration to the Indies 15791600, The Americas, 33, pp. 78-95.

Boyd-Bowman Peter (1976b) Patterns of Spanish Emigration to the Indies until 1600, The Hispanic American Historical Review, 56, pp. 580-604.

Boyd-Bowman Peter (1976c) Spanish Emigrants to the Indies, 1595-98: A Profile, in Fredi Chiappelli Ed., First Images of America, the Impact of the New World on the Old, vol. 2, Berkeley, University of California Press, pp. 723-36.

Bullard Alice (2000) Exile to Paradise: Savagery and Civilization in Paris and the South Pacific, 1790-1900, Stanford, Stanford University Press, 392 p. 
Campbell Persia Crawford (1923) Chinese Coolie Emigration to Countries within the British Empire, London, P.S. King \& Son, 240 p.

Campbell Gwyn, Miers Suzanne and Miller Joseph C. Eds (2007) Women and Slavery, Vol. 1 and 2, Athens, Ohio University Press, 404 p.

Cañizares-Esguerra, Jorge and Seeman Erik Eds (2007) The Atlantic in Global History: 1500-2000, Upper Saddle River, Prentice-Hall, 258 p.

Castles Stephen and Miller Mark J. (2009) The Age of Migration: International Population Movements in the Modern World (4th edition), New York, Guilford Press, $392 \mathrm{p}$.

Cohen Lucy M. (1971)The Chinese of the Panama Railroad: Preliminary Notes on the Migrants of 1854 who “Failed", Ethnohistory, 18, pp. 309-320.

Curtin Philip (1990) The Rise and Fall of the Plantation Complex: Essays in Atlantic History, New York, Cambridge University Press, 1990, 222 p.

Damousi Joy (1997) Depraved and Disorderly: Female Convicts, Sexuality and Gender in Colonial Australia, Cambridge, Cambridge University Press, 221 p.

Davis Nira Yuval (1997) Gender and Nation, London, Sage Publications, 168 p.

Dejohn Anderson Virginia (1985) Migrants and Motives: Religion and the Settlement of New England, 1630-1640, The New England Quarterly, 58, pp. 339-383.

Donato Katharine et al. (2011) Variations in the Gender Composition of Immigrant Populations: How They Matter, International Migration Review, 45, pp. 495-526.

Ekirch A. Roger (1987) The Transportation of British Convicts to the Colonies, 1718- 1775, New York, Oxford University Press, 277 p.

Eltis David and Engerman Stanley L. (1992) Was the Slave Trade Dominated by Men?, The Journal of Interdisciplinary History, 23, pp. 237-257.

Eltis David (1983) Free and CoercedTransatlantic Migrations: Some Comparisons, The American Historical Review, 88, pp. 251-280.

Foner Eric (1995) Free Soil, Free Labor, Free Men:The Ideology of the Republican Party Before the Civil War, New York, Oxford University Press, 400 p.

Gabaccia Donna R. (1996) Women of the Mass Migrations: From Minority to Majority, 1820-1930, in Dirk Hoerder and Leslie Page Moch Eds., European Migrants: Global and Local Perspectives, Boston, Northeastern University Press, pp. 90-111.

Gabaccia Donna R. (1997)The "Yellow Peril" and the "Chinese of Europe": Global Perspectives on Race and Labor, 1815-1930, in Jan and Leo Lucassen Eds., Migrations, Migration History, History: Old Paradigms and New Perspectives, Berlin, Peter Lang, pp. 76-96.

Gabaccia Donna R. (2000) Italy's Many Diasporas: Elites, Exiles and Workers of the World, London, University College of London Press, 264 p.

Gabaccia Donna R. (2001) When the Migrants Are Men:Women, Transnationalism and Italian Family Economies, in Pam Sharpe Ed., Women and Labor Migration: Global and Historical Perspectives, London, Routledge Press, pp. 190-208. 
Gabaccia Donna R. (2004) A Longer Atlantic in a Wider World, Atlantic Studies, 1, pp. 1-27.

Gabaccia Donna R. and Hoerder Dirk Eds. (2011) Connecting Seas and Connected Ocean Rims: Indian, Atlantic and Pacific Oceans and China Seas Migrations from the 1830s to the 1930s, Leiden, Brill Academic Publishers, 552 p.

Gabaccia Donna R. and Donato Katharine (2015) Gender and International Migration: From the Slavery Era to the Global Age, New York, Russell Sage Foundation, $270 \mathrm{p}$.

Games Alison (1999) Migration and the Origins of the English Atlantic World, Harvard Historical Studies, 133, Cambridge, Harvard University Press, 325 p.

Geggus David (1989) Sex Ratio, Age and Ethnicity in the Atlantic Slave Trade: Data from French Shipping and Plantation Records, The Journal of African History, 30, pp. 23-44.

Grubb Farley (1994) The End of European Immigrant Servitude in the United States: An Economic Analysis of Market Collapse, 1772-1835, The Journal of Economic History, 54, pp. 794-824.

Grubb Farley (2000) The Transatlantic Market for British Convict Labor, The Journal of Economic History, 60, pp. 94-122.

Guasco Michael (2010) Abolition of Slavery, New York, Oxford University Press, $34 \mathrm{p}$.

Gutman Herbert G. (1976) The Black Family in Slavery and Freedom, 1750-1925, New York, Pantheon Books, 664 p.

Guttentag Marcia and Secord Paul (1983) Too Many Women? The Sex Ratio Question, Beverly Hills, Sage Publications, 277 p.

Haines Robin (1994) Indigent Misfits or Shrewd Operators? GovernmentAssisted Emigrants from the United Kingdom to Australia, 1831-1860, Population Studies, 48, pp. 223-247.

Hammerton James (1979) Emigrant Gentlewomen: Genteel Poverty and Female Emigration, 1830-1914, Canberra, Australian National University Press, 220 p.

Harzig Christiane and Hoerder Dirk (2009) What is Migration History?, Cambridge, Polity Press, $200 \mathrm{p}$.

Herzfeld Michael (2004) Cultural Intimacy: Social Poetics in the Nation-State, New York and London, Routledge, 280 p.

Hoerder Dirk (1999) From Immigration to Migration Systems: New Concepts in Migration History, OAH, Magazine of History, 14, pp. 5-12.

Hoerder Dirk (2002) Cultures in Contact: World Migrations in the Second Millenium, Durham, Duke University Press, 808 p.

Hoerder Dirk (2013) Transcultural Approaches to Gendered Labour Migrations: From the Nineteenth-Century Proletarian to the Twenty-first-Century Caregiver Mass Migrations, in Dirk Hoerder and Amarjit Kaur Eds., Proletarian and Gendered Mass Migrations: A Global Perspective on Continuities and Discontinuities from the 19th to the 21st Centuries Studies in Global Social History and Studies in Global Migration History, Leiden, Brill Publishers, pp. 65-84. 
Hoerder Dirk and Kaur Amarjit (2013) Proletarian and Gendered Mass Migrations. A Global Perspective on Continuities and Discontinuities from the 19th to the 21st Centuries, Brill, [online] Books and Journals, collection Social Sciences E-Books Online, Book DOI: 10.1163/9789004251380

Hsu Madeline (2000) Dreaming of Gold, Dreaming of Home: Transnationalism and Migration Between the United States and South China, 1882-1943, Stanford, Stanford University Press, 320 p.

Hughes Sarah S. (1994) Gender at the Base of World History, The History Teacher, 27, pp. 417-423.

Jain Shobhita et Reddock Rhoda Eds (1998) Women Plantation Workers: International Experiences, New York, Berg, 186 p.

King Russell, Skeldon Ronald and Vullnetari Julie (2008) Internal and International Migration: Bridging the Theoretical Divide, IMISCOE 'Theories of Migration and Social Change Conference', St. Anne's College, Oxford, 1-3 July 2008, [online] last checked 29/07/2013. URL : http://www.imi.ox.ac.uk/pdfs/russell-king-ronskeldon-and-julie-vullnetari-internal-and-international-migration-bridging-thetheoretical-divide

Klein Martin A. (2007) Sex, Power, and Family Life in the Harem: A comparative Study, in Gwyn Campbell, Suzanne Miers and Joseph C. Miller Eds., Women and Slavery, Vol. 1: Africa, the Indian Ocean World and the Medieval North Atlantic, Athens, Ohio University Press, pp. 63-82.

Kupperman Karen Ordahl (2012) The Atlantic in the World, New York, Oxford, $166 \mathrm{p}$.

Lai Walton Look (1993) Indentured Labor, Caribbean Sugar: Chinese and Indian Migrants to the British West Indies, 1818-1918, Baltimore and London, Johns Hopkins University Press, 370 p.

Lee Sharon M. (1989) Female Immigrants and Labor in Colonial Malaya: 18601947, International Migration Review, 23, pp. 309-331.

Lopez Kathleen (2013) Chinese Cubans: A Transnational History, Chapel Hill, University of North Carolina Press, 339 p.

Lorde Audre (1984) Sister Outsider: Essays and Speeches, Berkeley, Crossing Press, $256 \mathrm{p}$.

Lovejoy Paul and Richardson David (1995) Competing Markets for Male and Female Slaves: Prices in the Interior of West Africa, 1780-1850, The International Journal of African Historical Studies, 28, pp. 261-293.

Lovejoy Paul (2007) Internal Markets or an Atlantic-Sahara Divide? How Women Fit into the Slave Trade of West Africa, in Gwyn Campbell, Suzanne Miers and Joseph C. Miller Eds., Women and Slavery, Vol. 1: Africa, the Indian Ocean World and the Medieval North Atlantic, Athens, Ohio University Press, pp. 259-280.

Lucassen Jan and Lucassen Leo Eds (1997) Migrations, Migration History, History: Old Paradigms and New Perspectives, Berlin, Peter Lang, 454 p.

Lucassen Jan and Lucassen Leo (2009) The Mobility Transition Revisited, 15001900: What the Case of Europe Can Offer to Global History, Journal of Global History, 4 (3), pp. 347-377. 
Manning Patrick (1996) Slave Trades: 1500-1800: Globalization of Forced Labour, Aldershot, Variorum, $361 \mathrm{p}$.

Manning Patrick (2004) Migration in World History, London, Routledge, 220 p.

Massey Doreen B. (1994) Space, Place and Gender, Minneapolis, University of Minnesota Press, 280 p.

Matt Susan J. (2011) Homesickness: An American History, New York, Oxford University Press, $360 \mathrm{p}$.

McDonald John and Richards Eric (1997) The Great Emigration of 1841: Recruitment for New South Wales in British Emigration Fields, Population Studies, 51, pp. 337-355.

McKeown Adam (2001) Chinese Migrant Networks and Cultural Change: Peru, Chicago, Hawaii, 1900-1936, Chicago, University of Chicago Press, 349 p.

McKeown Adam (2004) Global Migration, 1846-1940, Journal of World History, $15,155-89$.

McKeown Adam (2008) Melancholy Order: Asian Migration and the Globalization of Borders, New York, Columbia University Press, 450 p.

McKeown Adam (2010) Chinese Emigration in Global Context, 1850-1940, Journal of Global History, 5, pp. 95-124.

Meagher Arnold J. (2003) The Coolie Trade: The Traffic in Chinese Laborers to Latin America, 1847-1874, Philadelphia, Xlibris, 486 p.

Miller Joseph C. (1988) Way of Death: Merchant Capitalism and the Angolan Slave Trade 1730-1830, Madison, University of Wisconsin Press, 692 p.

Moller Herbert (1945) Sex Composition and Correlated Culture Patterns of Colonial America, The William and Mary Quarterly, 3rd Series, 2, pp. 113-153.

Morgan Gwenda and Rushton Peter (2004) Eighteenth-Century Criminal Transportation: The Formation of the Criminal Atlantic, New York, Palgrave Macmillan, $238 \mathrm{p}$.

Morgan Jennifer (2004) Laboring Women: Reproduction and Gender in New World Slavery, Philadelphia, University of Pennsylvania Press, 296 p.

Morokvasic Mirjana (1984) Birds of Passage are also Women, International Migration Review, 18, pp. 886-907.

Northrup David (1995) Indentured Labor in the Age of Imperialism, 1834-1922, New York, Cambridge University Press, $186 \mathrm{p}$.

Nwokeji G. Ugo (2001) African Concepts of Gender and the Slave Traffic, William and Mary Quarterly, 3rd series, 58, pp. 47-68.

Nwokeji G. Ugo (2010) The Slave Trade and Culture in the Bight of Biafra: An African Society in the Atlantic World, Cambridge, Cambridge University Press, $256 \mathrm{p}$.

O'Reilly William (2012) Movements of People in the Atlantic World, 1450-1850, in Nicholas Canny and Philip Morgan Eds., The Oxford Handbook of the Atlantic World: 1450-1850, Oxford University Press, Oxford Handbooks, [online] DOI: 10.1093/oxfordhb/9780199210879.001.0001 
O'Rourke Kevin H. and Williamson Jeffrey G. (1999) Globalization and History: The Evolution of a Nineteenth-Century Atlantic Economy, Cambridge, MIT Press, $343 \mathrm{p}$.

Oxley Deborah (1996) Convict Maids: The Forced Migration of Women to Australia, Cambridge, Cambridge University Press, 339 p.

Peck Gunther (2000) Reinventing Free Labor: Padrones and Immigrant Workers in the North American West, 1880-1930, Cambridge, Cambridge University Press, $332 \mathrm{p}$.

Pessar Patricia R. and Mahler Sarah (2001) Gendered Geographies of Power: Analyzing Gender across Transnational Spaces, Identities: Global Studies in Culture and Power, 7, pp. 441- 59.

Ramirez Bruno (1991) On The Move: French-Canadian and Italian Migrants in the North Atlantic Economy, 1861-1914, Toronto, McClelland and Stewart Publishing, $174 \mathrm{p}$.

Ravenstein E.G. (1876) The Birthplace of the People and the Laws of Migration, The Geographical Magazine, 3, pp. 173-177, 201-206, 229-233.

Ravenstein E.G. (1885) The Laws of Migration, Journal of the Statistical Society of London, 48, pp.167-235.

Ravenstein E.G. (1889) The Laws of Migration, Journal of the Royal Statistical Society, 52, pp. 241-305.

Robertson Robbie (2003) The Three Waves of Globalization: A History of a Developing Global Consciousness, London, Zed Books, 281 p.

Robson Leslie Lloyd (1994) The convict settlers of Australia (2nd ed), Melbourne, Melbourne University Press, 229 p.

Samaroo Brinsley (2013) Indian and Chinese Immigration to the British Caribbean, in Immanuel Ness Ed., Encyclopedia of the History of Global Migrations, Oxford, Wiley-Blackwell.

Schrover Marlou (2013) Feminization and Problematization of Migration: Europe in the Nineteenth andTwentieth Centuries, in Dirk Hoerder and Amarjit Kaur Eds., Proletarian and Gendered Mass Migrations, Leiden, Brill Publishers, pp. 103-31.

Seaver Kirsten A. (2007) Thralls and Queens: Female Slavery in the Medieval Norse Atlantic, in Gwyn Campbell, Suzanne Miers and Joseph C. Miller Eds., Women and Slavery, Vol. 1: Africa, the Indian Ocean World and the Medieval North Atlantic, Athens, Ohio University Press, pp. 147-168.

Sen Samita (2004) Without His Consent? Marriage and Women's Migration in Colonial India, International Labor and Working-Class History, 65, pp. 77-104.

Shammas Carole (2013) Household Formation, Lineage, and Gender Relations in the Early Modern Atlantic World, in Nicholas Canny and Philip Morgan Eds., The Oxford Handbook of the Atlantic World: 1450-1850, Oxford, Oxford University Press, [online] DOI: 10.1093/oxfordhb/9780199210879.001.0001

Silvey Rachel and Lawson Victoria (1999) Placing the Migrant, Annals of the Association of American Geographers, 89, pp. 121-132.

Silvey Rachel (2006) Geographies of Gender and Migration: Spatializing Social Difference, International Migration Review, 40, pp. 64-81. 
Sinke Suzanne M. (2006) Gender and Migration: Historical Perspectives, International Migration Review, 40, pp. 82-103.

Sinn Elizabeth Yuk Yee (2013) Pacific Crossing: California Gold, Chinese Migration, and the Making of Hong Kong, Hong Kong, Hong Kong University Press, 454 p.

Steinfeld Robert J. (1991) The Invention of Free Labor: The Employment Relation in English and American Law and Culture, 135-1870, Chapel Hill, University of North Carolina Press, 286 p.

Steward Watt (1951) Chinese Bondage in Peru: A History of Chinese Coolies in Peru, 1849-1874, Durham, Duke University Press, [online] http://babel.hathitrust. org/cgi/pt?id=mdp.39015027242026;view=1up;seq=11

Tinker Hugh (1974) A New System of Slavery: The Export of Indian Labour Overseas, 1830-1920, London, Oxford University Press, 432 p.

United Found for Population Activities (UNFPA) (2006) A Passage to Hope: Women and International Migration. State of the World's Population 2006, New York, UNFPA.

Van Ruymbeke Bertrand (2008) L'histoire Atlantique aux États-Unis : la périphérie au centre, Nuevo Mundo Mundos Nuevos, [en ligne] consulté le 24/04/2015. URL : http://nuevomundo.revues.org/42083; DOI : 10.4000/nuevomundo.42083

Weevers Marian H.A.C. (2012) Swept Up from the Streets or Nowhere Else to Go?The Journeys of Dutch Female Beggars and Vagrants to the Oegstgeest State Labor Institution in the Late Nineteenth Century, Journal of Social History, 46, pp. 416-429.

Westmarland Nicole (2001) The Quantitative/Qualitative Debate and Feminist Research: A Subjective View of Objectivity, Forum: qualitative social research, 2, Art. 13.

Wiesner-Hanks Merry (2007) World History and the History of Women, Gender, and Sexuality, Journal of World History, 18, pp. 53-67.

Willcox Walter and Ferenczi Imre Eds (1970) International Migrations, 2 vols., Publications of the National Bureau of Economic Research, 14, New York, Arno Press, [online] http://www.nber.org/chapters/c5101.pdf.

Williamson Jeffrey G. (1996) Globalization, Convergence and History, The Journal of Economic History, 56, pp. 277-306

Zlotnik Hania (2003) Data Insight: The Global Dimensions of Female Migration, Migration Information Source, [online] http://www.migrationinformation.org/ feature/display.cfm?ID109 


\section{Donna R. Gabaccia}

\section{Genre et migrations dans les études atlantiques de 1500 à nos jours}

Malgré les efforts pour décrire les connexions entre l'Atlantique et le reste du monde, les chercheurs ne s'accordent pas sur la périodisation de l'intégration mondiale et la fin du statut de l'Atlantique comme une macro-région particulière. Ils reconnaissent toutefois que les flux migratoires massifs sont un élément constitutif de ce processus et certains chercheurs affirment que l'intégration mondiale récente a produit la féminisation des mouvements migratoires dans toutes les régions du monde. Dans cet article, l'auteure s'intéresse à la question du genre dans les migrations internationales, depuis les traites négrières jusqu'au début du XXe siècle. Elle montre que la féminisation des migrations commence avant le XXe siècle et que le processus s'est poursuivi au cours des années 1960.

\section{Spatializing Gender and Migration: the Periodization of Atlantic Studies, 1500-present}

Despite recent efforts to describe the changing relationship of the Atlantic to the wider world, scholars do not agree on the periodization of global integration and the end of the Atlantic's status as a unique macro-region. They do, however, agree that mass migratory flows are a constitutive element of global integration; some social sciences argue further that recent global integration has produced the feminization of migratory movements across all world regions. This paper traces the gender composition of long distance migrations from the early modern slave trades to the twentieth century. It finds convergence in gendered patterns of migration beginning already in the early twentieth century and argues that feminization of international migrations was complete by 1960 .

\section{Género y migraciones en los estudios atlánticos del año 1500 a nuestros días}

A pesar de los esfuerzos por describir las conexiones entre el Atlántico y el resto del mundo los investigadores no logran ponerse de acuerdo sobre la periodización de la integración mundial y el fin del estatus del Atlántico como una macro-región particular. Reconocen, no obstante, que los flujos migratorios masivos son un elemento constitutivo de este proceso y ciertos investigadores afirman que la integración mundial reciente produjo la feminización de los movimientos migratorios en todas las regiones del mundo. En este artículo, la autora se interesa por la cuestión de género en las migraciones internacionales, desde las tratas negreras hasta principios del siglo XX. Muestra que la feminización de las migraciones comienza antes del siglo $X X$ y que el proceso continuó en el transcurso de los años sesenta. 